\title{
Synthesis and Characterization of Stearic Acid-Beclomethasone Dipropionate Conjugates with the Potential for Improving Loading Capacity in Lipid-based Nanoparticles
}

Shishuai Dang ${ }^{1, \#}$, Zhengwei Huang ${ }^{1,2, \#}$, Ying Huang ${ }^{2, *}$, Xin Pan ${ }^{1}$, Chuanbin Wu ${ }^{1,2}$

1 School of Pharmaceutical Sciences, Sun Yat-sen University, Guangzhou, 510006, China

2 College of Pharmacy, Jinan University, Guangzhou 510632, P. R. China

\# S. Dang and Z. Huang contributed equally to this work.

* To whom correspondence should be addressed.

Tel: +86 02039943115 (Ying Huang, Ph.D.)

Fax: +86 02039943115 (Ying Huang, Ph.D.)

Email: huangy2007@163.com (Ying Huang, Ph.D.)

\section{ABSTRACT}

\section{Purpose}

This study aims to provide a potential technology platform to improve the drug encapsulation ability of low lipophilicity drugs in Lipid-based nanoparticles (LBNs), and broaden the application of LBNs in pulmonary delivery.

\section{Methods}

A proof-of-concept was carried out using Beclomethasone dipropionate (BDP) as a model drug. Herein, BDP was conjugated with stearic acid (SA), a kind of the lipid matrix for LBNs. By this means, the intermolecular interactions between the cargo and the lipid matrix can be enhanced. The conjugate was characterized by nuclear magnetic resonance (NMR), infrared (IR), and X-ray diffraction (XRD) spectra. The interactions between the conjugate and SA were investigated by molecular dynamics simulation, and compared with the interactions between BDP and SA.

\section{Results}

The molecular dynamics simulation proved that modification can improve the lipophilicity of the drug and enhance the interaction between the drug and the lipid material through structural similarity.

\section{Conclusion}

This study anticipates establishing a technology platform to improve the loading capacity of low lipophilicity drugs in LBNs, for the therapy of lung diseases. It is expected that the drug loading capacity of weak-lipophilic drugs in LBN can be increased by establishing the technology platform, and the application of LBNs in pulmonary delivery can be broadened.

\section{KEYWORDS}

Lipid-based nanoparticles, beclomethasone dipropionate, stearic acid, pulmonary

administration 


\section{INTRODUCTION}

Recently, nanoparticulate sustained release systems like lipid-based nanoparticles (LBNs) have been attracting increasing interest. LBNs are drug delivery systems based on surfactantsstabilized lipid matrices, including solid lipid nanoparticle (SLN), nanostructured lipid carriers (NLC), polymer-lipid hybrid nanoparticle (PLN) and liposome, etc. LBNs are versatile systems, and the application of LBN in the pulmonary drug delivery region has gradually shown broad prospects.

In pulmonary administration, the irritation of the drug delivery system to the respiratory tract should always be considered during the formulation design. LBNs are composed of highly biocompatible lipid materials, which can significantly reduce the irritation to the respiratory tract (Leifer et al. 2018). This allows LBNs to fit the pulmonary drug delivery system well (Puri et al. 2009). At the same time, because the frequency of pulmonary drug administration greatly affects the patient compliance, reducing the administration frequency through sustained-release technology has become an important research hotspot. From this stand point, as LBNs have an outstanding sustained-release effect(Zielinska et al. 2020), they enable to reduce the frequency administration, resulting in improved patient compliance, which is essential in the pulmonary administration therapy(Rehman et al. 2018). These attributes account for the popularity of LBNs in the field of pulmonary drug delivery.

However, there remain an important hurdle for the wider application of LBNs in pulmonary delivery. Many active pharmaceutical ingredients for pulmonary disease treatment are of low lipophilicity, including ambroxol hydrochloride, terbutaline sulfate salbutamol sulfate and beclomethasone dipropionate (BDP). These low lipophilic drugs will show low encapsulation capacity in LBNs that consists of lipid materials(Triolo et al. 2017), due to 'Like dissolves like' principles. This in turns causes that the dosage should be raised or the dosing frequency should be increased. In this case, the aim of improving patient compliance cannot be achieved.

In response to this problem, this study anticipated establishing a technology platform that is for improving the loading capacity of drugs with low lipophilicity in LBNs, for pulmonary administration. A preliminary proof-of-concept study was carried out using BDP as a model drug.

In order to improve the loading capacity of BDP in LBNs, in this study, proper modification of BDP was performed based on the "Like dissolves like" principle. Stearic acid (SA) is one of the generally used lipid matrices for LBN, which has the advantages of good biocompatibility. A recent study even shows that elevated production of the antiinflammatory cytokine interleukin-10 (IL-10) has been detected in stearic acid-treated hepatocytes(Nishitani et al. 2007), which implies that stearic acid is capable of the alleviation of inflammation. The modification by SA will expect low mucosal irritation and no toxic side effects in pulmonary delivery.

Therefore, BDP was conjugated with SA to be lipid-drug conjugate (LDC) called stearic acid-beclomethasone dipropionate conjugates (SA-BDP). With this method, the intensity of the interactions between the cargo and the lipid matrices are strengthened due to the similar structure. By a stronger molecular interaction with the LBNs lipid matrix, the encapsulation capacity of BDP in LBNs could be enhanced. This study may provide a potential technology 
platform to improve the drug encapsulation ability of low lipophilicity drugs in LBNs, and broaden the application of LBNs in pulmonary delivery.

\section{MATERIALS AND METHODS}

\subsection{Materials}

Subheadings should be typeset in boldface italics, and capitalize the first letter of the first word only. Section number to be in boldface Roman. BDP was purchased from YuanYe BioTechnology Co., Ltd. (Shanghai, China). The silica gel (200 300 mesh) for column chromatography and silica gel GF254 for thin layer chromatography (TLC) were purchased from Lige Technology Co., Ltd. (Guangzhou, China). Stearoyl chloride, acetonitrile, methylene chloride, Boron fluoride ethyl ether, petroleum ether and ethyl acetate were purchase from Aladdin Bio-Chem Technology Co., Ltd. (Shanghai, China).

\subsection{Methods}

\subsubsection{SA-BDP preparation}

Synthesis. Figure 1 displayed the synthesis pathway for preparing SA-BDP. All glass instruments and pipette tips are dried in a drying oven in advance for $24 \mathrm{~h}$. Precisely $100 \mathrm{mg}$ of BDP was weighed and dissolved in $4 \mathrm{~mL}$ of $\mathrm{CH}_{2} \mathrm{Cl}_{2} / \mathrm{MeCN}(50 / 50, \mathrm{v} / \mathrm{v})$. Then, $100 \mu \mathrm{l}$ of stearoyl chloride was added slowly. After complete dissolution, $100 \mu \mathrm{l}$ of $\mathrm{BF}_{3} \cdot \mathrm{Et}_{2} \mathrm{O}$ was added. Keep the reaction liquid and water isolated throughout. Reactions were carried out at $0^{\circ} \mathrm{C}$ for $2 \mathrm{~h}$ followed by $1 \mathrm{~h}$ of room temperature. The reaction was monitored with TLC.

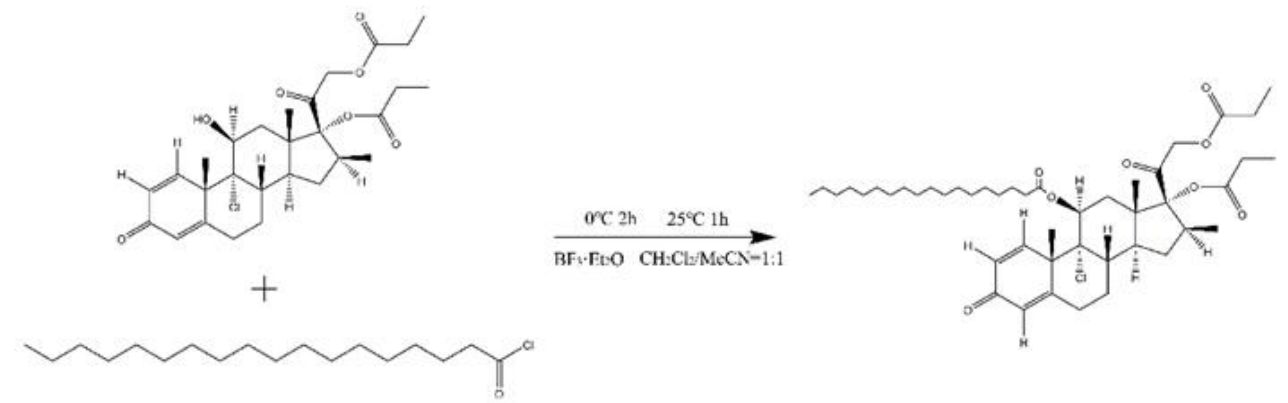

Fig.1 The synthetic route of SA-BDP

Purifications. Solvents were removed under vacuum to complete dryness in a speed-vacuum dryer. It was then redissolved in $2 \mathrm{ml}$ petroleum ether and ethyl acetate $(5: 1, \mathrm{v} / \mathrm{v})$. Purification was performed using silica gel column chromatography with an elution volume of $50 \sim 70 \mathrm{ml}$ (eluent system: petroleum ether: ethyl acetate $=5: 1, \mathrm{v} / \mathrm{v}$ ). The separation was monitored by TLC analysis. After high vacuum drying, $\sim 30 \mathrm{mg}$ of the product was obtained.

\subsubsection{Characterization}

Nuclear magnetic resonance (NMR). ${ }^{1} \mathrm{H}-\mathrm{NMR}$ spectra were obtained with a NMR spectrometer (Bruker, AVANCE III HD 400M, Germany) with spectrometer frequency offset of the first(observe) channel in $400.1324708 \mathrm{MHz}$. Spectra were obtained at a temperature of $25^{\circ} \mathrm{C}$, using zg30 pulse sequences, $10 \mu$ s pulse width, 8196.722 spectral width, and the number of sampling points was 65536 . The relaxation duration was $1 \mathrm{~s}$. Number of scans was 16. The time domain data were apodized with an exponential window function using a line broadening of $0.3 \mathrm{~Hz}$. 
${ }^{13} \mathrm{C}$-NMR spectra were obtained with a Bruker AVANCE III HD 400M spectrometer with spectrometer frequency offset of the first (observe) channel in $100.6228298 \mathrm{MHz}$. Spectra were obtained at a temperature of $25^{\circ} \mathrm{C}$, using zgpg30 pulse sequences, $10 \mu$ s pulse width, 23809.523 spectral width, and the number of sampling points was 65536 . The relaxation duration was $2 \mathrm{~s}$. Number of scans was 1024 . The time domain data were apodized with an exponential window function using a line broadening of $1.0 \mathrm{~Hz}$.

Fourier Transform Infrared (IR). For FTIR analysis, $2 \mathrm{mg}$ dried BDP/SA-BDP was properly mixed with $200 \mathrm{mg}$ potassium bromide $(\mathrm{KBr})$ and compressed to prepare a disc of about $3 \mathrm{~mm}$ in diameter. FTIR spectroscopy of the disc was recorded FTIR spectrometer (Thermo, Nicolet iS10, USA) at room temperature, in the frequency range of 400 to $4000 \mathrm{~cm}^{-1}, 32$ scans per sample at a resolution of $4 \mathrm{~cm}^{-1}$.

The X-ray diffraction (XRD). XRD pattern was recorded using an X-ray diffractometer (Rigaku, D8, Japan). The experimental parameters of the XRD experiment on the BDP/SABDP were as follows: A copper target $(45 \mathrm{kV} \times 200 \mathrm{~mA})$ was used at room temperature, $2 \times$ $106 \mathrm{cps}$ of the linear range of the scintillation counter and a step scanning method for data collection. Qualitative analysis using Bruker DIFFRAC.EVA V4.3 and refinement using Bruker DIFFRAC.TOPAS V6.0.

\subsubsection{Molecular dynamics simulation}

As the purpose of this work was to enhance BDP encapsulation efficiency in LBNs, the molecular dynamics simulation method was used to predict the drug-loading capacity of LBNs to SA-BDP. SA-BDP shared a similar structure with SA (the lipid matrices of LBNs), and it was anticipated that the interactions between SA-BDP and SA would be significantly stronger than that between BDP and SA. Figure 2 displayed the molecular structures of SA and SA-BDP, and the identical alkyl (C18) chain was highlighted.

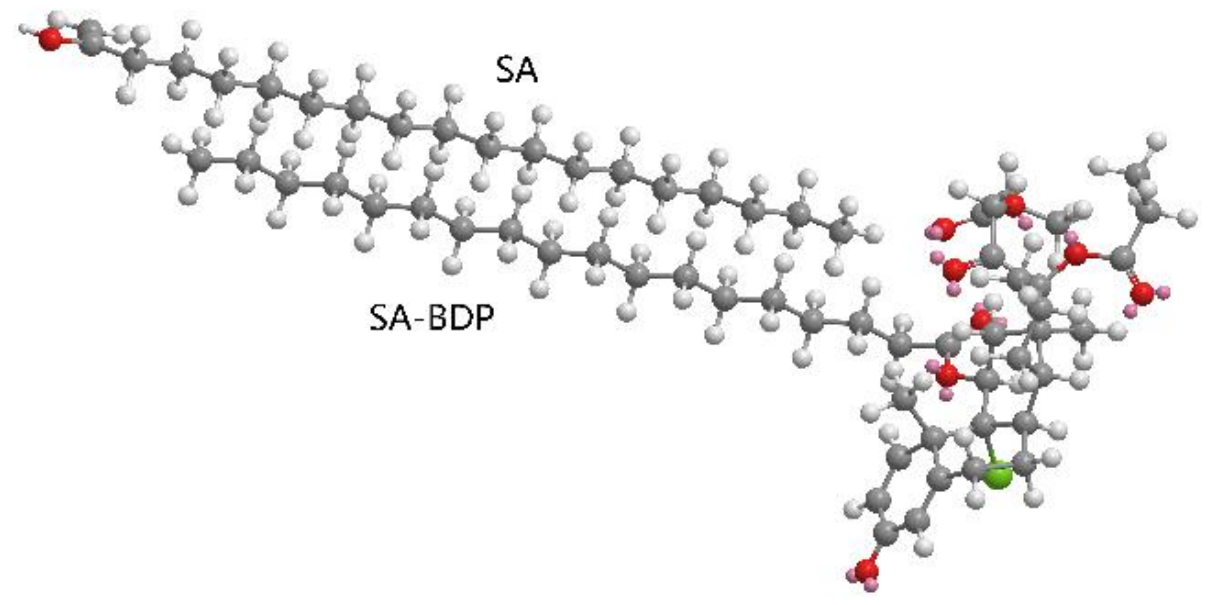


In this study, the electrostatic force and van der Waals force were investigated. The reasons were as follows. The electrostatic interaction had a tuning effect on the stability of particles in solution and guides the self-assembly of the secondary structure. Van der Waals force was a weak interaction that generally existed among all molecules or atoms. These two interactions would help to figure out the interactions between SA and SA-BDP, and between SA and BDP. Simulations for the electrostatic force and the van der Waals force between SABDP and SA were carried out using Gromacs-4.6.7 and compared with the electrostatic force between BDP and SA.

All the all-atom MD simulations were based on a GROMOS54a7 force field(Schmid et al. 2011) created by ATB(Malde et al. 2011) and were carried out using the Gromacs-4.6.7 software package(Hess et al. 2008). We used the relaxed system as a starting configuration. As it is prior to system relaxation MD, energy minimization was carried out with a composite protocol of steepest descent using termination gradients of $100 \mathrm{~kJ} / \mathrm{mol} \cdot \mathrm{nm}$. A $10 \mathrm{~ns} \mathrm{NPT}$ relaxation run at $298 \mathrm{~K}$ for the equilibrium MD simulation. The Nose'-Hoover thermostat(Berendsen et al. 1984) was used to maintain the equilibrium temperature at $298 \mathrm{~K}$ and periodic boundary conditions were imposed on all three dimensions. The Particle MeshEwald method(Astrakas et al. 2012; Essmann et al. 1995)was used to compute long-range electrostatics within a relative tolerance of $1 \times 10-6$. A cut-off distance of $1 \mathrm{~nm}$ was applied to real-space Ewald interactions. The same value was used for van der Waals interactions. The LINCS algorithm(Hess et al. 2008) was applied to constrain bond lengths of hydrogen atoms. A leap-frog algorithm(Van Gunsteren and Berendsen 1988) was used with a time step of $2 \mathrm{fs}$.

\section{RESULTS}

Among the numerous lipid materials, SA is one of the most popular materials applied in sustained release drug delivery(Lerata et al. 2020). Our goal was to obtain a modified product of BDP that had high drug loading capacity if encapsulated in SA-based LBNs. For this purpose, BDP was conjugated with SA through a substitution reaction. This modification can improve the lipophilicity of the drug and enhance the interaction between the drug and the lipid material through structural similarity.

\subsection{SA-BDP preparation}

The synthesis of SA-BDP was accomplished in a one-step reaction. This was similar to the reaction Radek Gazak et al. completed on silybin(Gazak et al. 2010). Interestingly, in that study, silybin had multiple hydroxyl groups and easily produced by-products, resulting in lower purity of the product. While in this study, BDP has only one hydroxyl group, and the reaction with SA would exhibit high selectivity and the amount of by-products might be neglectable. The yield of the reaction is $14 \sim 27 \%(\mathrm{w} / \mathrm{w})$. Compared with many other LDC synthesis methods(Ding et al. 2018; Olbrich et al. 2002), the reaction does not require heating, and has great potential in practical applications, particularly for the thermolabile drugs.

In preliminary study, a variety of separation methods have been considered, like silica gel column chromatography, dextran gel column, molecular sieve chromatography, thin-layer plate separation and extraction. Due to the poor water solubility of the reactants and products, and the susceptibility to acid/alkali degradation, the silica gel column chromatography separation was chosen. It was a method with high separation ability and easy operation, which has been widely used in the pharmaceutical industry(Jin et al. 2019). Compared with commonly used vacuum distillation, vacuum drying can effectively prevent the drug from degradation under heat, remove the solvent more thoroughly, and is easier for transferring. 


\subsection{Characterization}

We report the structural characterization of BDP (as reference) and SA-BDP. The molecular and crystal structures of both compounds are reported and discussed.

\subsubsection{NMR}

Figure 3A D displayed the ${ }^{1} \mathrm{H}$ NMR spectra and ${ }^{13} \mathrm{C}$ NMR spectra of BDP and SA-BDP. The chemical shifts in NMR spectra were listed below.
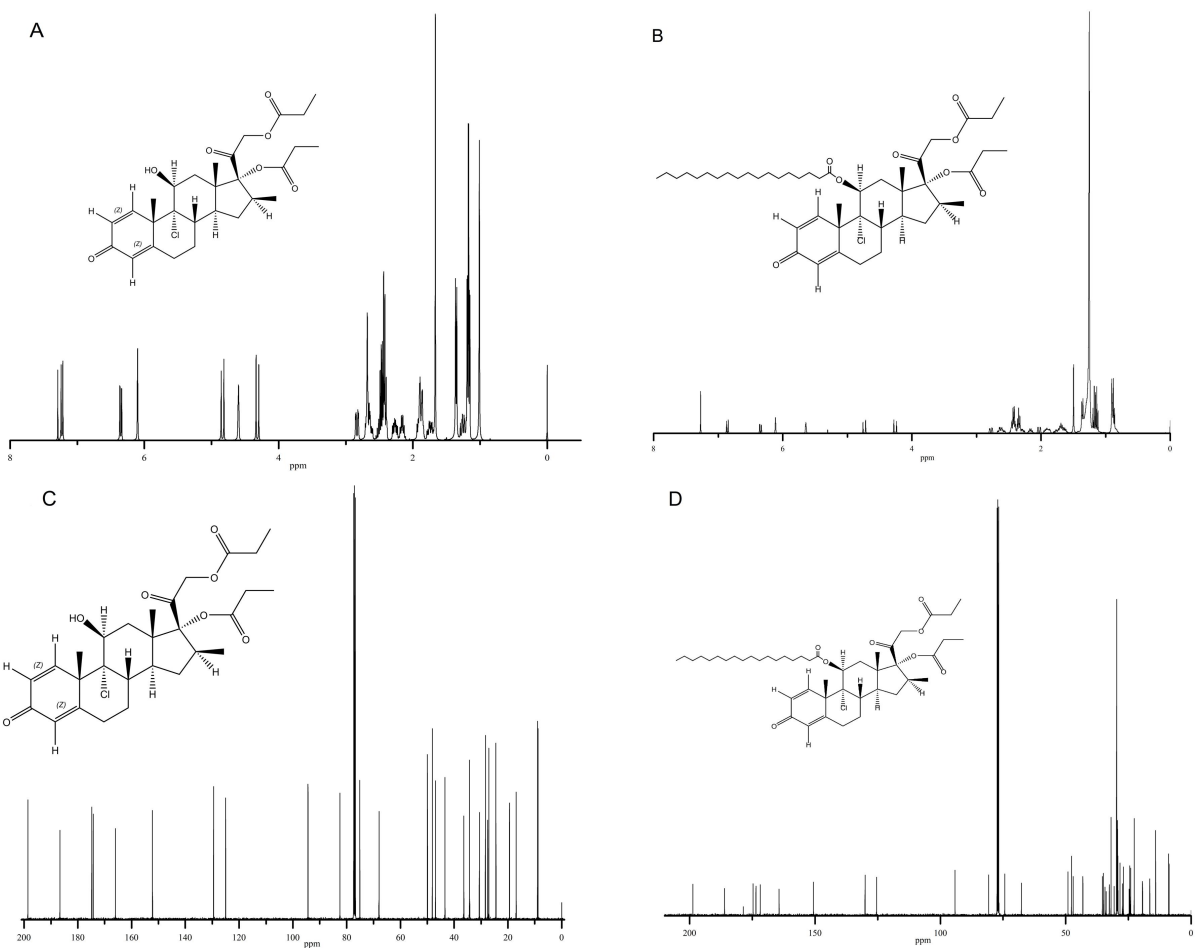

Fig.3 The ${ }^{1} \mathrm{H}-\mathrm{NMR}$ spectra of BDP (A) and SA-BDP (B), and the ${ }^{13} \mathrm{C}-\mathrm{NMR}$ of BDP (C) and SA-BDP (D)

BDP ${ }^{1} \mathrm{H}$ NMR (400 MHz, Chloroform- $d$ ) $\delta=7.23(\mathrm{~d}, J=10.1 \mathrm{~Hz}, 1 \mathrm{H}), 6.35$ (dd, $J=10.1$, $1.8 \mathrm{~Hz}, 1 \mathrm{H}), 6.10(\mathrm{~s}, 1 \mathrm{H}), 4.84(\mathrm{~d}, J=16.4 \mathrm{~Hz}, 1 \mathrm{H}), 4.60(\mathrm{~s}, 1 \mathrm{H}), 4.32(\mathrm{~d}, J=16.4 \mathrm{~Hz}, 1 \mathrm{H})$, $2.83(\mathrm{dd}, J=14.1,3.6 \mathrm{~Hz}, 1 \mathrm{H}), 2.78-2.57(\mathrm{~m}, 3 \mathrm{H}), 2.56-2.34(\mathrm{~m}, 5 \mathrm{H}), 2.34-2.09(\mathrm{~m}, 2 \mathrm{H})$, $1.99-1.83(\mathrm{~m}, 3 \mathrm{H}), 1.75(\mathrm{~m}, J=13.5,11.7,5.5 \mathrm{~Hz}, 1 \mathrm{H}), 1.36(\mathrm{~d}, J=7.3 \mathrm{~Hz}, 3 \mathrm{H}), 1.30-1.22$ $(\mathrm{m}, 1 \mathrm{H}), 1.17(\mathrm{~m}, J=7.5,2.8 \mathrm{~Hz}, 6 \mathrm{H}), 1.01(\mathrm{~s}, 3 \mathrm{H})$.

BDP ${ }^{13} \mathrm{C}$ NMR $\left(100 \mathrm{MHz}, \mathrm{CDCl}_{3}\right): \delta=198.5,186.6,174.8,174.2,165.9,152.2,129.4$, 125.0, 94.3, 82.4, 82.4, 77.2, 75.1, 67.9, 49.9, 48.1, 46.9, 43.4, 36.4, 34.2, 34.2, 30.6, 28.3, $27.5,27.1,24.4,19.4,16.9,8.9,8.7$.

SA-BDP ${ }^{1} \mathrm{H}$ NMR $(400 \mathrm{MHz}$, Chloroform- $d): \delta=6.86(\mathrm{~d}, J=10.1 \mathrm{~Hz}, 1 \mathrm{H}), 6.34(\mathrm{dd}, J=$ $10.1,1.8 \mathrm{~Hz}, 1 \mathrm{H}), 6.11(\mathrm{~s}, 1 \mathrm{H}), 5.64(\mathrm{~s}, 1 \mathrm{H}), 4.74(\mathrm{~d}, J=16.4 \mathrm{~Hz}, 1 \mathrm{H}), 4.26(\mathrm{~d}, J=16.3 \mathrm{~Hz}$, $1 \mathrm{H}), 2.78(\mathrm{dd}, J=14.4,3.7 \mathrm{~Hz}, 1 \mathrm{H}), 2.62(\mathrm{~m}, J=18.4,12.6,12.0,5.4 \mathrm{~Hz}, 2 \mathrm{H}), 2.52-2.24$ (m, $8 \mathrm{H}), 2.16(\mathrm{q}, J=8.0 \mathrm{~Hz}, 1 \mathrm{H}), 2.03(\mathrm{dd}, J=14.4,2.6 \mathrm{~Hz}, 1 \mathrm{H}), 1.91(\mathrm{~m}, J=18.4,15.4,5.5 \mathrm{~Hz}$, $2 \mathrm{H}), 1.83-1.58(\mathrm{~m}, 4 \mathrm{H}), 1.50(\mathrm{~s}, 3 \mathrm{H}), 1.36(\mathrm{~d}, J=7.4 \mathrm{~Hz}, 3 \mathrm{H}), 1.16(\mathrm{~m}, J=15.4,7.5 \mathrm{~Hz}, 6 \mathrm{H})$, $0.89(\mathrm{dd}, J=11.6,5.3 \mathrm{~Hz}, 8 \mathrm{H})$.

SA-BDP ${ }^{13} \mathrm{C}$ NMR $\left(100 \mathrm{MHz}, \mathrm{CDCl}_{3}\right): \delta=198.7,186.0,178.6,174.6,173.5,171.8,164.3$, 150.6, 130.0, 125.4, 94.1, 80.7, 77.2, 74.3, 67.6, 49.0, 47.7, 47.0, 43.1, 35.3, 34.9, 34.3, 33.9, 

$24.7,24.4,24.1,22.7,19.3,16.4,14.1,8.9,8.8$.

The main difference between the NMR spectra of BDP and SA-BDP was that after the modification of the SA fatty chain, the addition of hydrocarbyl hydrogen significantly reduces the original hydrogen intensity, but its position is basically unchanged, which indicates the success of the modification.

\subsubsection{IR spectra}

Figure $4 \mathrm{~A}$ and $\mathrm{B}$ summarized the IR spectra of BDP and SA-BDP.
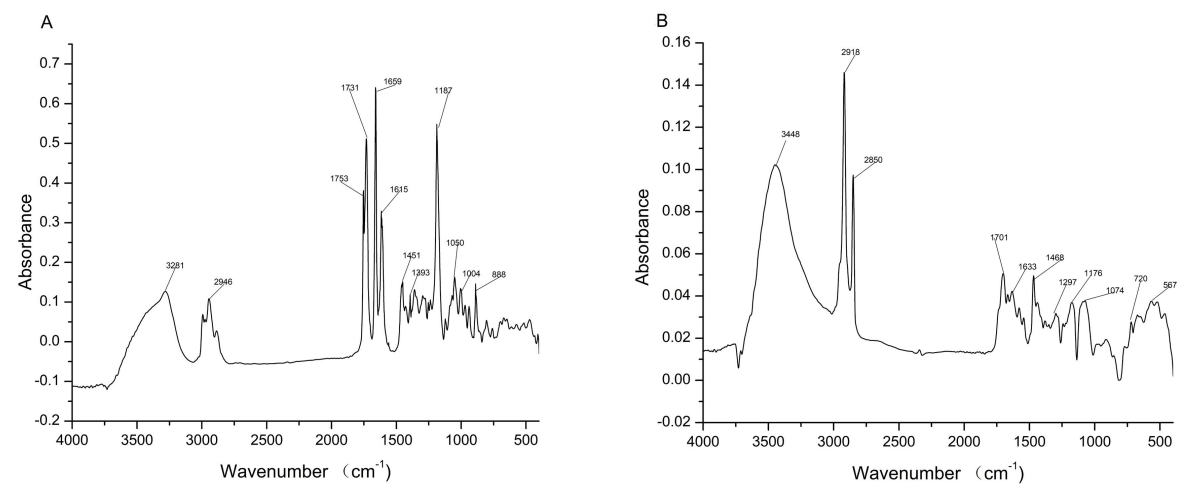

Fig.4 The IR spectra of BDP (A) and SA-BDP (B)

FT-IR analysis confirmed the presence of characteristic functional groups from BDP. The absorption at $3281 \mathrm{~cm}^{-1}$ was clearly due to the stretching vibration of the $(-\mathrm{OH})$ groups, Meanwhile, the two absorption bands at $2946 \mathrm{~cm}^{-1}$ were observed, which were assigned to the antisymmetric and symmetric stretching vibration of the $\mathrm{C}-\mathrm{H}$ in methyl or methylene. The absorption at $3010 \mathrm{~cm}^{-1}$ was due to the stretching vibration of the $=\mathrm{C}-\mathrm{H}$, the absorptions at $939 \mathrm{~cm}^{-1}$ and $888 \mathrm{~cm}^{-1}$ were due to the wagging vibration of the $=\mathrm{C}-\mathrm{H}$, the absorption at 1753 $\mathrm{cm}^{-1}$ was due to the stretching vibration of the unconjugated $\mathrm{C}=\mathrm{O}$ groups, the absorption at $1731 \mathrm{~cm}^{-1}$ was due to the stretching vibration of the $\mathrm{C}=\mathrm{O}$ groups, the absorptions at $1615 \mathrm{~cm}^{-1}$ and $1659 \mathrm{~cm}^{-1}$ were due to the stretching vibration of the $\mathrm{C}=\mathrm{C}$, the absorptions at $1451 \mathrm{~cm}^{-1}$ and $1393 \mathrm{~cm}^{-1}$ was due to the bending vibration of the $\mathrm{C}-\mathrm{H}$, the absorption at $1187 \mathrm{~cm}^{-1}$ was due to the stretching vibration of the $\mathrm{C}-\mathrm{O}$ in ester group, and the absorptions at $1004 \mathrm{~cm}^{-1}$ and $1050 \mathrm{~cm}^{-1}$ were due to the stretching vibration of the $\mathrm{C}-\mathrm{O}-\mathrm{C}$ in ester group.

The similar features in the IR spectra of the SA-BDP also showed the characteristic peaks. The two absorption bands at $2918 \mathrm{~cm}^{-1}$ and $2850 \mathrm{~cm}^{-1}$ were observed, which were assigned to the antisymmetric and symmetric stretching vibration of the $\mathrm{C}-\mathrm{H}$ in methyl or methylene. The absorptions at $3010 \mathrm{~cm}^{-1}$ was due to the stretching vibration of the $=\mathrm{C}-\mathrm{H}$, and the absorptions at $1297 \mathrm{~cm}^{-1}$ and $1468 \mathrm{~cm}^{-1}$ were due to the bending vibration of the $\mathrm{C}-\mathrm{H}$, the absorption at $1701 \mathrm{~cm}^{-1}$ was due to the stretching vibration of the $\mathrm{C}=\mathrm{O}$ groups, the absorption at $1633 \mathrm{~cm}^{-1}$ was due to the stretching vibration of the $\mathrm{C}=\mathrm{C}$, the absorption at $1176 \mathrm{~cm}^{-1}$ was due to the stretching vibration of the $\mathrm{C}-\mathrm{O}$ in ester group, the absorption at $1074 \mathrm{~cm}^{-1}$ was due to the stretching vibration of the $\mathrm{C}-\mathrm{O}-\mathrm{C}$ in ester group, and the absorption at $720 \mathrm{~cm}^{-1}$ was due to the wagging vibration of the $\mathrm{C}-\mathrm{H}$, which showed there were long chain alkyl groups in the sample.

The main difference between IR spectra of BDP and SA-BDP was the $720 \mathrm{~cm}^{-1}$ peak appeared in the SA-BDP spectra, which was due to the wagging vibration of the $\mathrm{C}-\mathrm{H}$. This showed there were long chain alkyl groups in the sample. 
Figure 5A and $\mathrm{B}$ showed the XRD spectra of BDP and SA-BDP. BDP showed many characteristics peaks, which indicates that there are many crystal orientations; while SA-BDP simple, indicating that SA-BDP is easier to form a single crystal. This might be attributed to the different lattice structures of BDP and SA-BDP.

Simulated XRD patterns were calculated with Bruker DIFFRAC.TOPAS V6.0 and compared with the experimentally observed pattern. Figure 5C and D showed the XRD refinement spectra of BDP and SA-BDP: Fit data (red), test Data (blue) and difference (green). The test data is consistent with the fitted data, R-factor of weighted profile (Rwp) $<10 \%$, indicating that the crystalline structures of BDP and SA-BDP are consistent with the prediction.
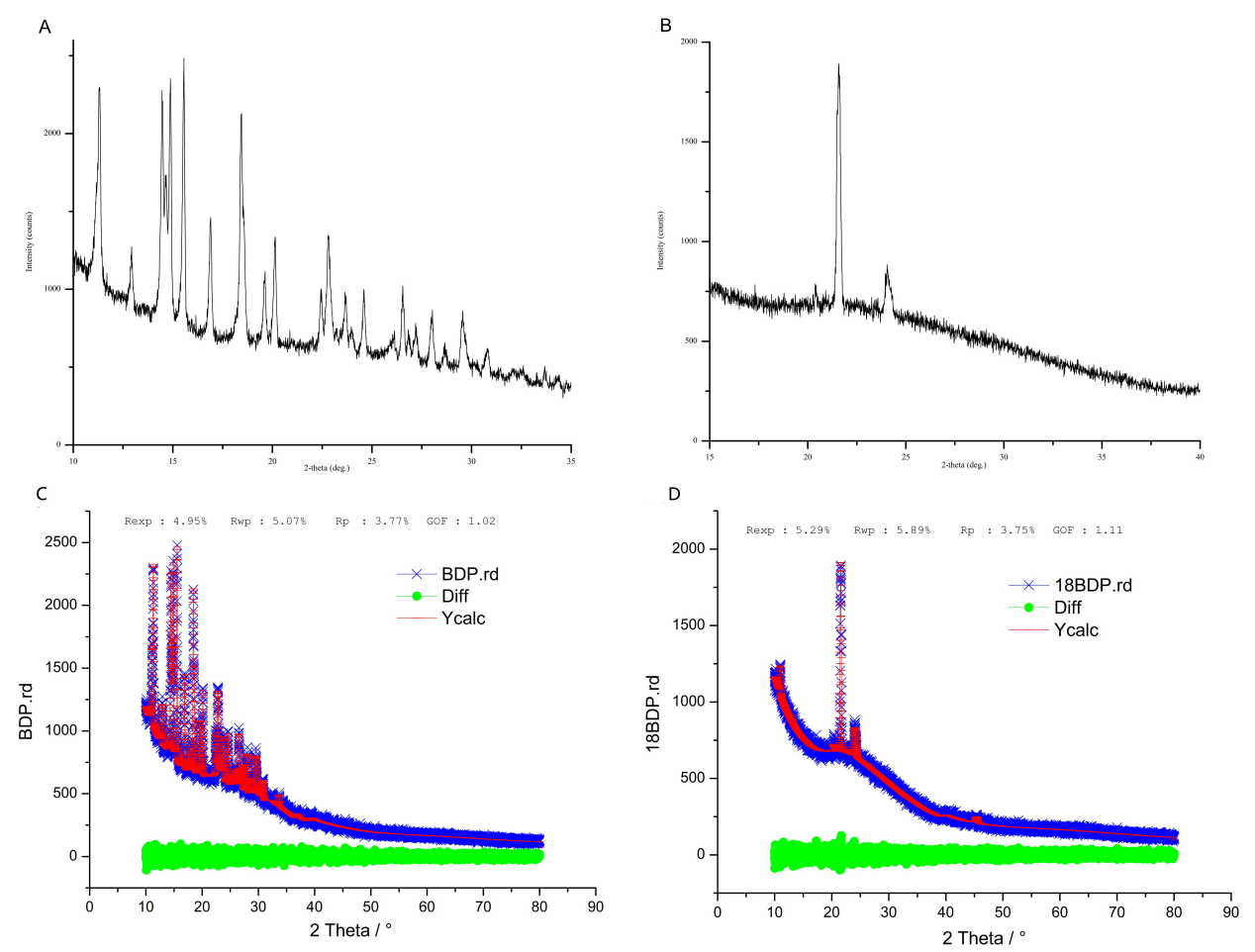

Fig.5 The XRD spectra of BDP (A) and SA-BDP (B) and the XRD refinement spectra of BDP (C) and SA-BDP (D): Fit data (red), Test Data (blue), Difference (green)

By far, the differences in the NMR, IR and XRD spectra of BDP and SA-BDP demonstrated that SA-BDP was successfully synthesized, and the related spectroscopic behaviors of SA-BDP were significantly different from BDP.

\subsection{Molecular dynamics simulation}

\subsubsection{Electrostatic force}

Figure 6A showed the time evolution of the electrostatic force between two molecules in a water system.

It can be seen from Figure 6A that the electrostatic force between the two systems basically shows a dynamic balance, and the electrostatic forces of the two systems are relatively close. The electrostatic force depends on the size of the dipole moment of the polar group, the degree of orientation, the distance between the molecules and the ambient temperature. The strength of the electrostatic force between the lipid matrix and the drug 
before and after modification is around $40 \mathrm{~kJ} / \mathrm{mol}$, which may be related to the small dipole moment of the lipid matrix and the limited modification of the drug's dipole moment.

\subsubsection{Van der Waals force}

Figure 6B showed how the time evolution of the van der Waals force between two molecules in a water system.
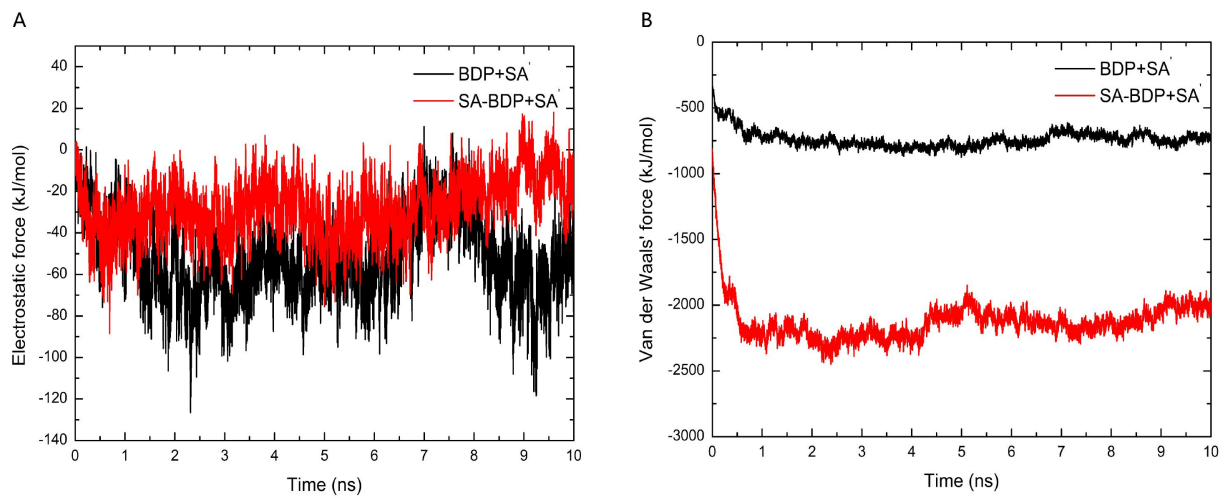

Fig.6 The electrostatic force (A) and the Van der Waals force (B) between molecules changes with the simulation time of SA-BDP + SA (black) and BDP + SA (red)

It can be seen from Figure 6B that the van der Waals interaction force between the two systems basically shows a dynamic balance, and the van der Waals interaction force between the two systems differs greatly. What needs illustration is that the larger the absolute value of electrostatic interaction or van der Waals interaction, the stronger the force. Therefore, the total interaction between SA-BDP and SA molecules is stronger than that between BDP and SA molecules. The van der Waals force between the modified drug and the lipid matrix is almost three times that before the modification. In addition to the principle of "Like dissolves like", this is due to the more dispersed electron cloud distribution of the modified drug and the increase in the intermolecular interaction area, which increases the dispersion force.

In summary, it is theoretically speculated that the electrostatic interaction between SABDP and SA was similar to that between BDP and SA, but the van der Waals force between SA-BDP and SA was stronger than that between BDP and SA. Therefore, SA-BDP and SA have stronger intermolecular interactions than BDP and SA. Due to the stronger molecular interaction between SA-BDP and SA, it can be predicted that the loading capacity of SA-BDP in LBNs will be desirable. Besides, the burst release effect of SA-BDP loaded LBNs might also be reduced. Further researches will verify the real loading situation.

\section{DISCUSSION}

In order to establish a technology platform to improve the loading capacity of drugs with undesirable lipophilicity in LBNs, we explored the feasibility of SA modification using BDP as a model drug, and finally showed that this modification is feasible and is expected to improve the loading capacity, a study on nicotine oral delivery also indirectly implies it(Ding et al. 2018). In the future, we will expand the target drugs to ambroxol hydrochloride, terbutaline sulfate and salbutamol sulfate, etc. (these drugs for pulmonary disease therapy all have low lipophilicity), as they also contain hydroxyl groups in the molecular structure and the similar substitution reaction can be performed. Actually, due to the generality of hydroxyl groups in drug molecules, this technology platform possesses high potential for application. 
Based on BDP model, this study can not only build a technical platform, but also bring potential opportunities for asthma treatment. Because as the first-line treatment for asthma(Reddel et al. 2019), the recommended dosage of BDP is $800 \mu \mathrm{g}$ bid, and long-term prophylactic administration is required. A large number of studies have proved that frequent administration of drugs reduces the patient compliance and thus affects the treatment effect(Aggarwal et al. 2018; Juniper 2003; Plaza et al. 2018). After improving the loading efficiency of BDP in LBNs, the frequency of administration can be significantly reduced, and it will directly reduce the impact of long-term treatment on the patient's quality of life and avoid the psychological burden of the "patient" stereotype on the patient. From this perspective, this work may finally improve patient compliance and thus have a positive impact on the treatment of asthma.

Because of the long alkyl chain $\left(\mathrm{C}_{18}\right)$ structure of SA, the SA-BDP conjugate may even be able to self-assemble into a nanocrystalline drug delivery system without relying on external lipids(Ivanova et al. 2017), and the feasibility of its self-assembly will be explored in the future.

\section{CONCLUSION}

This study anticipates establishing a technology platform to improve the loading capacity of low lipophilicity drugs in LBNs, for the therapy of lung diseases. It is expected that the drug loading capacity of weak-lipophilic drugs in LBN can be increased by establishing the technology platform, and the application of LBNs in pulmonary delivery can be broadened.

\section{ACKNOWLEDGMENTS}

This work was supported by the [Special Funds for the Cultivation of Guangdong College Students' Scientific and Technological Innovation ("Climbing Program" Special Funds)] under Grant [number pdjh2019a0003]; and [National Natural Science Foundation of China] under Grant [number 81673375; 81703431]; The authors wish to thank Zhen Yang (RWTH Aachen University) and Hui Wang (Sun Yat-sen University) for their kind help in the synthetic experiments.

\section{COMPLIANCE WITH ETHICAL STANDARDS}

\section{Conflict of Interest}

The authors declare that they have no conflict of interest.

\section{Statement of Human and Animal Rights}

This article does not contain any studies with human and animal subjects performed by any of the authors.

\section{REFERENCES}

Aggarwal AN, Kumari R,Grover S (2018) Patient Satisfaction With Inhaled Medication for Asthma. Respir Care 63: 859-864.

Astrakas LG, Gousias C,Tzaphlidou MJJoAP (2012) Structural destabilization of chignolin under the influence of oscillating electric fields. 111: 074702. 
Berendsen HJC, et al. (1984) Molecular dynamics with coupling to an external bath. The Journal of Chemical Physics 81: 3684-3690.

Ding Y, et al. (2018) Lipid-drug-conjugate (LDC) solid lipid nanoparticles (SLN) for the delivery of nicotine to the oral cavity - Optimization of nicotine loading efficiency. Eur J Pharm Biopharm 128: 10-17.

Essmann U, et al. (1995) A smooth particle mesh Ewald method. 103: 8577-8570.

Gazak R, et al. (2010) Antioxidant and antiviral activities of silybin fatty acid conjugates. Eur J Med Chem 45: 1059-1067.

Hess B, et al. (2008) GROMACS 4: Algorithms for Highly Efficient, Load-Balanced, and Scalable Molecular Simulation. J Chem Theory Comput 4: 435-447.

Ivanova EP, et al. (2017) Bactericidal activity of self-assembled palmitic and stearic fatty acid crystals on highly ordered pyrolytic graphite. Acta Biomaterialia 59: 148-157.

Jin N, et al. (2019) Maximum Loaded Amorphous Azithromycin Produced Using the Wetness Impregnation Method with Fractional Steps for Dermal Prophylaxis Against Lyme Disease. Die Pharmazie 74: 345-349.

Juniper EF (2003) The impact of patient compliance on effective asthma management. 9: S8S10.

Leifer FG, et al. (2018) Inhaled Treprostinil-Prodrug Lipid Nanoparticle Formulations Provide Long-Acting Pulmonary Vasodilation. Drug Res (Stuttg) 68: 605-614.

Lerata MS, et al. (2020) Encapsulation of Variabilin in Stearic Acid Solid Lipid Nanoparticles Enhances Its Anticancer Activity in Vitro. Molecules 25:

Malde AK, et al. (2011) An Automated Force Field Topology Builder (ATB) and Repository: Version 1.0. 7: 4026-4037.

Nishitani Y, et al. (2007) Saturated and monounsaturated fatty acids increase interleukin-10 production in rat hepatocytes. Nihon Arukoru Yakubutsu Igakkai Zasshi 42: 32-35.

Olbrich C, et al. (2002) Lipid-Drug-Conjugate (LDC) Nanoparticles as Novel Carrier System for the Hydrophilic Antitrypanosomal Drug Diminazenediaceturate. Journal of Drug Targeting 10: 387-396.

Plaza V, et al. (2018) Impact of patient satisfaction with his or her inhaler on adherence and asthma control. Allergy Asthma Proc 39: 437-444.

Puri A, et al. (2009) Lipid-Based Nanoparticles as Pharmaceutical Drug Carriers: From Concepts to Clinic. 26: 523-580.

Reddel HK, et al. (2019) GINA 2019: a fundamental change in asthma management. Treatment of asthma with short-acting bronchodilators alone is no longer recommended for adults and adolescents 53: 1901046.

Rehman A, Amin F,Sadeeqa S (2018) Prevalence of asthma and its management: A review. J Pak Med Assoc 68: 1823-1827.

Schmid N, et al. (2011) Definition and testing of the GROMOS force-field versions 54A7 and 54B7. 40: 843-856.

Triolo D, et al. (2017) Polymeric drug delivery micelle-like nanocarriers for pulmonary administration of beclomethasone dipropionate. Colloids and Surfaces B: Biointerfaces 151: 206-214.

Van Gunsteren WF,Berendsen HJCJMS (1988) A Leap-frog Algorithm for Stochastic Dynamics. 1: 173-185.

Zielinska A, et al. (2020) Loading, release profile and accelerated stability assessment of monoterpenes-loaded solid lipid nanoparticles (SLN). Pharm Dev Technol: 1-13. 
415

416
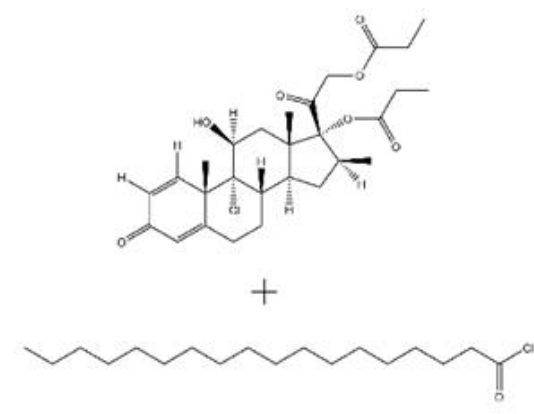

Fig.1 The synthetic route of SA-BDP

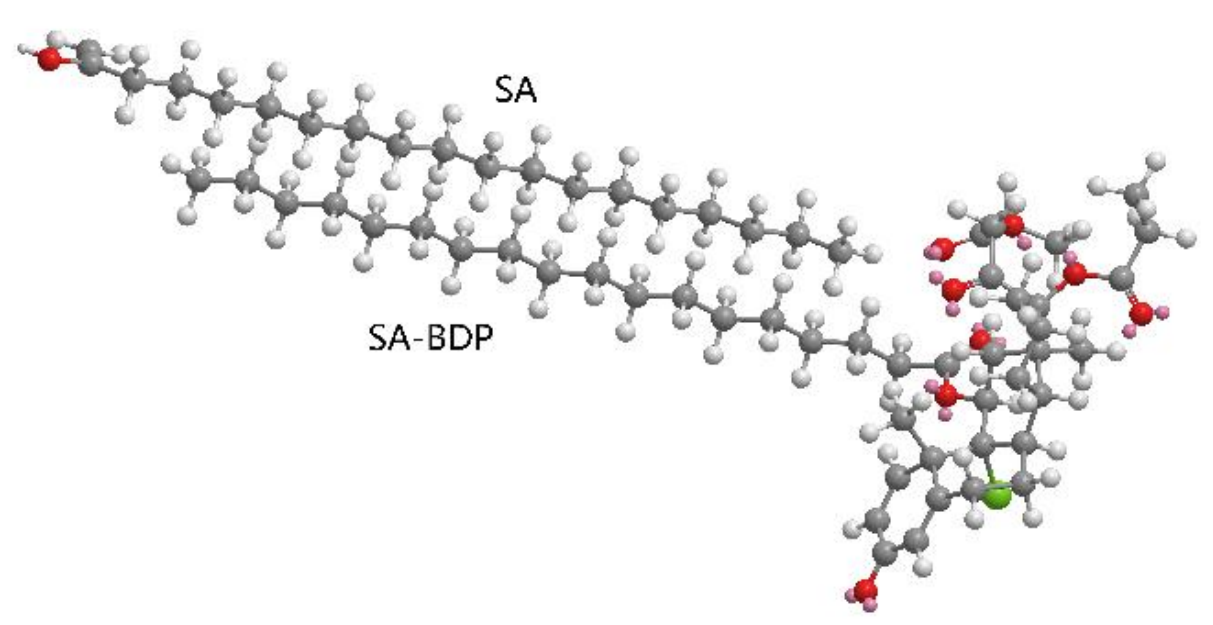

417

Fig.2 The molecular structures of SA and SA-BDP 

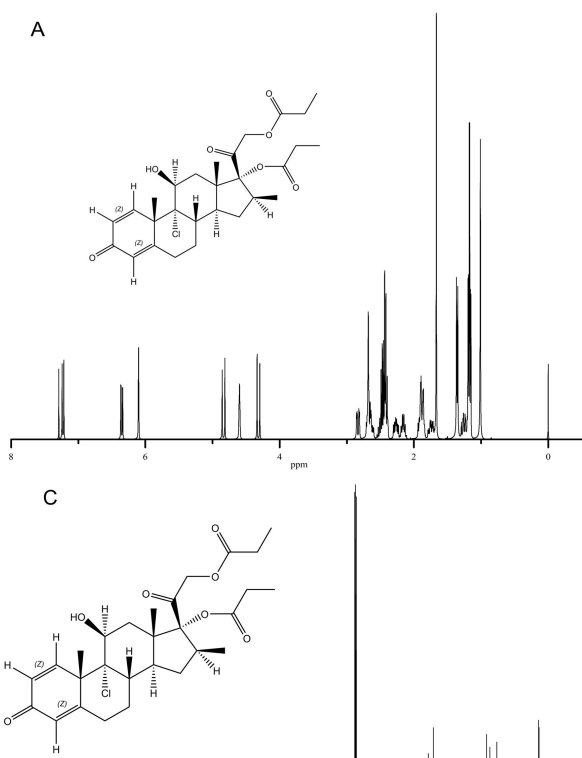

419 and SA-BDP (D)

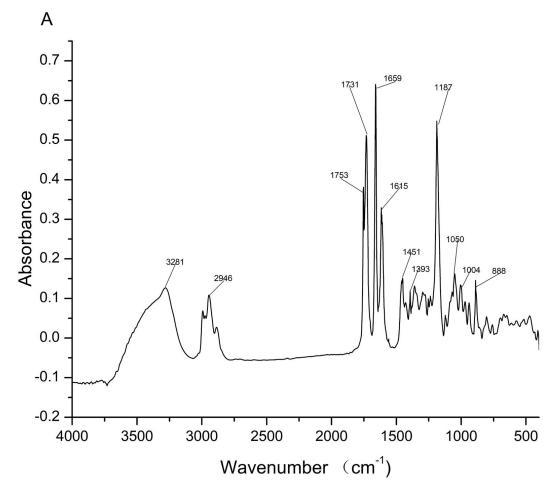

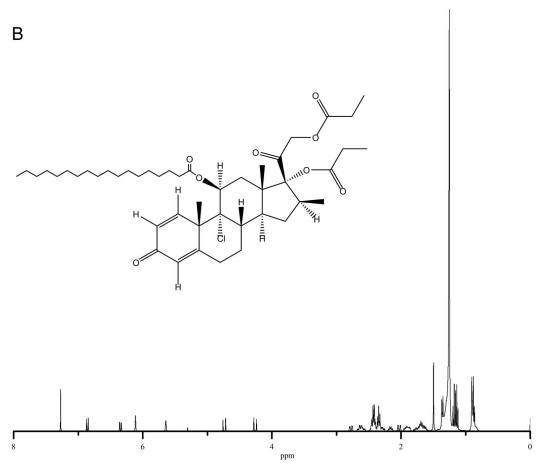

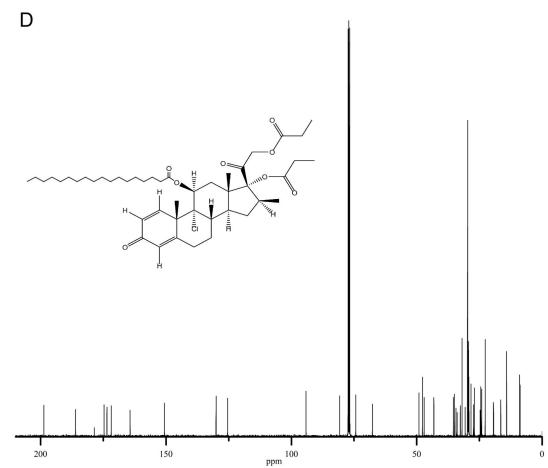

Fig. 3 The ${ }^{1} \mathrm{H}-\mathrm{NMR}$ spectra of BDP (A) and SA-BDP (B), and the ${ }^{13} \mathrm{C}-\mathrm{NMR}$ of BDP (C)

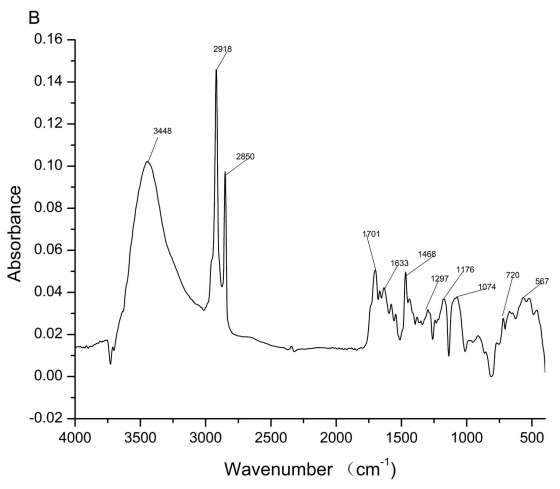

Fig.4 The IR spectra of BDP (A) and SA-BDP (B) 

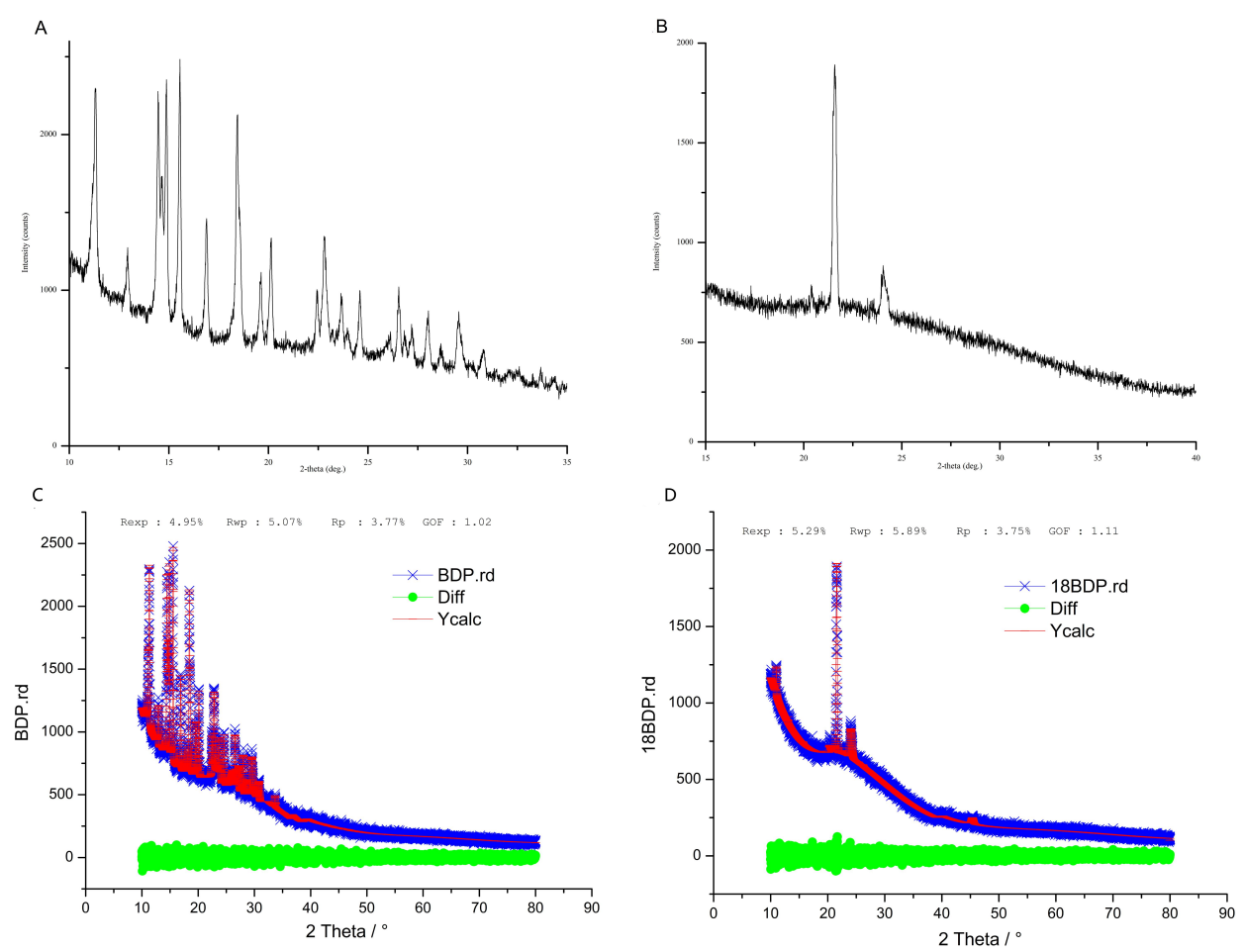

Fig.5 The XRD spectra of BDP (A) and SA-BDP (B) and the XRD refinement spectra of BDP (C) and SA-BDP (D): Fit data (red), Test Data (blue), Difference (green)
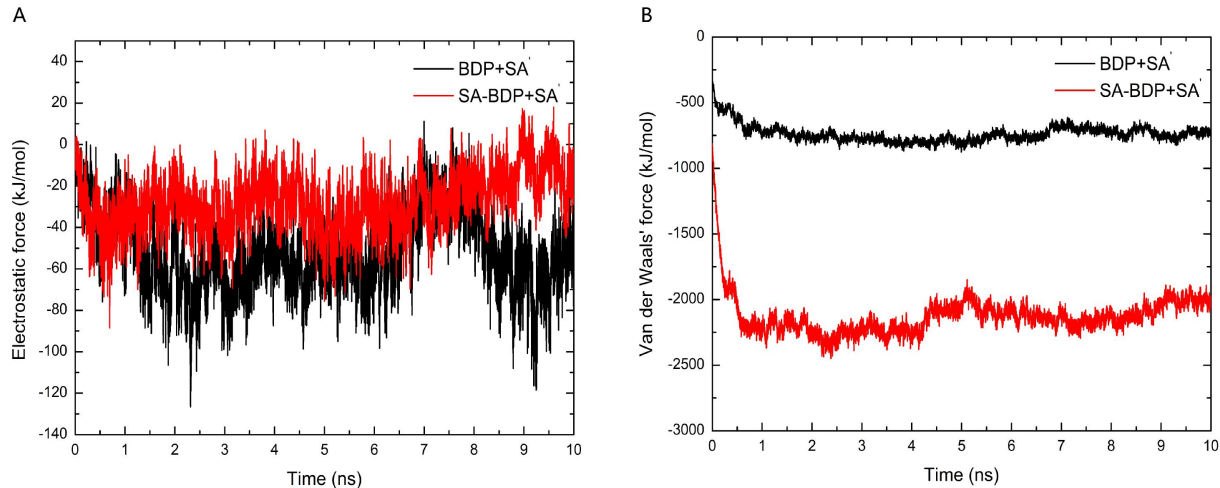

Fig.6 The electrostatic force (A) and the Van der Waals force (B) between molecules changes with the simulation time of SA-BDP + SA (black) and BDP + SA (red) 